\title{
A new MAC protocol of UASN (RABIC-MAC) based on high efficiency, concurrent and bidirection
}

\author{
LIU Jun ${ }^{1, a}$, YANG Xiao Lan ${ }^{2}$, PENG Ling Feng ${ }^{2}$ and LEI Peng Ying ${ }^{2}$ \\ ${ }^{1}$ School of Science, Chongqing University of Posts and Telecommunications, Chongqing, China, \\ 400065 \\ ${ }^{2}$ School of Optoelectronic Engineering, Chongqing University of Posts and Telecommunications, \\ Chongqing, China, 400065
}

Email ${ }^{\text {a }}$ liujun@cqupt.edu.cn

Keywords: UASN, throughput, end-to-end delay, MAC protocol

\begin{abstract}
Studies on MAC protocols of the Underwater Acoustic Sensor Network (UASN) are very important due to wide application area and great application potential of UASN. In this paper, a new concurrent bidirectional MAC protocol based on receiver self-adaption (RABIC-MAC) is proposed to improve the data transmission efficiency and reduce the overhead. Then the throughput and the End-to-End delay are calculated, and then compared with the BIC protocol. Results show that the saturated throughput is larger $16.7 \%$ and $33.0 \%$ for 4200 and $6125 \mathrm{~m}$, respectively, than the BIC. The saturated End-to-End delay is larger $13.0 \%$ and $20.0 \%$ for 4200 and $6125 \mathrm{~m}$, respectively, than the BIC. Therefore, the RABIC-MAC evidently improves performances of UASN.
\end{abstract}

\section{Introduction}

UWAN is very important in military, environment and energy source et al. Channel characteristics and performances of UWAN are evidently different from terrestrial wireless communication. Therefore, it is necessary to put forward new MAC protocols for UWAN. Current underwater MAC protocols mainly base on the fixed allocation mechanism and handshake mechanism [1, 2]. Compared with the fixed allocation mechanism, the handshake mechanism has many advantages such as alleviating node-hidden problems, carrying much information and reducing the collision between small controlling packets. On the other hand, UWAN may be affected by the uncertain space and time due to the high latency environment [3]. Traditional handshake mechanism often used three-way RTS/CTS/DATA, which resulted in the delay, and then low channel utilization [4]. To solve this problem, Chirdchoo et al proposed a three-way handshake protocol MACA-MN. Based on this protocol, data packet (DP) was transmitted to neighbors in a single handshake [5]. Soon they proposed a four-way handshake protocol RIPT, by which the receivers' initial reservation was utilized and the DP was gotten through coordinating multiple one-hops [6]. Liao et al proposed the protocol MR-MAC, by which three or more nodes were allowed to communicate in one handshake. By scheduling the packet transmission time, the DP was sent in a packet train manner and the receiver received DP without collision [7]. These handshaking-based MAC protocols often adopted unidirectional data transmission approach, which resulted in low channel utilization and large overhead. Recently, Ng et al proposed a concept ROPA, by which senders not only transmitted DP to receivers, but also got DP from one-neighbors [8]. Based on the ROPA, Yang et al proposed an improved protocol SRCR, by which a new concept 'concurrent' was added [9]. This means that senders and receiver may transmit DP to each other [10]. Although the bidirectional concurrent protocol BIC was proposed to allow senders and receivers exchange DP at the same time [10], it needed much exchanging time. Therefore, a new Receiver Self-adaption Bidirectional Concurrent protocol (RABIC) were proposed to shorten the exchanging time, and then solve packet collision problem. 


\section{Protocol Design}

Here it is assumed that each sensor is equipped with an omnidirectional half-duplex underwater acoustic modem. The propagation delay from a node to its one-hop neighbors and the maximum propagation delay from the one-hop neighbors to its neighbors are both given before the network initialization. The network initialization phases are given mainly via roundtrip time of controlling packets. Any estimated error and the flow fluctuation are very small. The small constant guard time ( $\left.\mathrm{T}_{\text {guard }}\right)$ is added to accommodate the error so that the error and the flow fluctuation may be omitted.

Some notations used in the RABIC-MAC protocol are defined as followed. $T_{D A T A}$ is the transmission time of each fixed-length DP. $T_{x}$ is the transmission time of the fixed-length controlling packet of type $x$, where $x \in\{\mathrm{RTS}, \mathrm{CTS}, \mathrm{NTF}\} . \tau_{\max }$ is the maximum propagation delay corresponding to the maximum transmission range. $\tau_{\max , i}$ is the maximum propagation delay between node $i$ and its one-hop neighbors. $\tau_{i, j}$ is the inter-nodal propagation delay between node $i$ and $j$. $S_{\text {burst }}$ is the threshold number of accumulated DPs for triggering a RTS attempt. $T_{\text {max }}$ is the time threshold for triggering a RTS attempt.$t_{r e f}$ is the starting time for exchanging bidirectional data of the S-R node pair. $d_{\text {silent, } i}^{x}$ is the duration when node $i$ remains silent after overhearing a type $x$ controlling packet, where $x \in\{\mathrm{RTS}$, CTS, NTF $\} . d_{\text {toc, } i}$ is the completion time, namely the duration when node $i$ is released from the handshake after $t_{\text {ref }} \cdot k_{s p \text { max }}$ is the maximum number of DPs transmitted in a special round to avoid TX-RX collisions. $k_{\max }$ is the maximum number of DPs transmitted in a CR to avoid TX-RX collisions. $n_{C R}$ is the total number of CRs. $d_{i}$ is the duration of a single $\mathrm{i} \in\{\mathrm{CR}, \mathrm{SP}, \mathrm{RR}\} . \quad n_{i}$ is the total number of relayed and new DPs. $n_{x, i}$ is the number of DPs corresponding type $x$, where $x \in$ \{relay, new $\}. N_{i}$ is the set of one-hop neighbor nodes of node $i$. $d_{\text {busy, } x}$ is the busy duration information carried within a type $x$ controlling packet, where $x \in\{$ RTS, CTS, NTF.$d_{\text {busy }, i}^{r x}$ is the busy duration receiving the last intended DP after $t_{\text {ref }}$ for node $i$. $k_{i}$ is the number of DPs transmitted either in a CR or RR by node $i . k_{s p}$ is the number of DPs transmitted in a special Round. Generally, the number of DPs transmitted from sender nodes to receiver nodes $n_{s}$ and that from receiver nodes to sender nodes $n_{R}$ should satisfy $n_{S} \geq n_{R}$ in a handshake. Based on the rule, there are three scenarios shown in Fig.1.

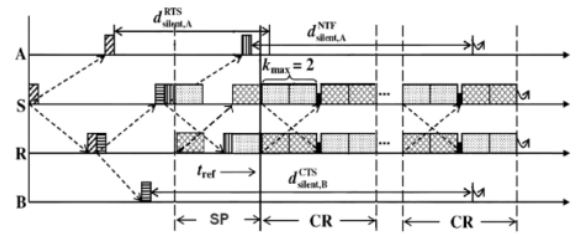

(a)

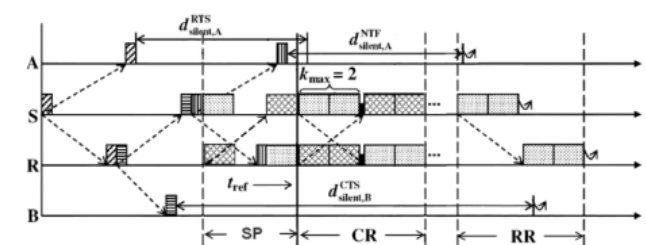

(b)

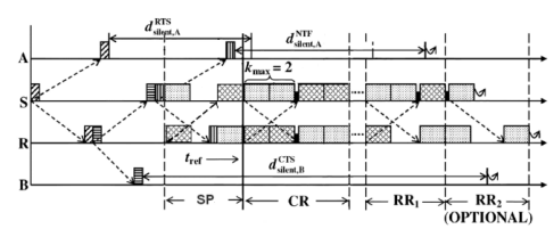

(c)

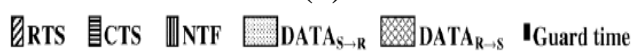

Fig.1. Time diagrams of RABIC-MAC: (a) type 1 scenario, (b) type 2 scenario and (c) type 3 scenario. Nodes ' $\mathrm{S}$ ' and ' $\mathrm{R}$ ' are sender and receiver, respectively. Nodes ' $\mathrm{A}$ ' and ' $\mathrm{B}$ ' are the one-hop neighbors of nodes ' $\mathrm{S}$ ' and ' $\mathrm{R}$ ', respectively.

The RABIC-MAC protocol mainly consists of two phases including Channel Reservation Phase and Bidirectional Concurrent Data Transmission Phase. These scenarios use the same exchange strategy of controlling packets. Here, an idle node uses a hybrid of batch-by-size and batch-by-time strategies to determine the time when the RTS are triggered. If trigger conditions are satisfied, the 
sender shall initiate its contention timer according to the Binary Exponential Backoff (BEB) algorithm, and then broadcasts his RTS to its one-hop neighbors to inform the total number of DPs transmitted for the current handshake. The transmitted DPs include their DP $\left(\mathrm{n}_{\text {new,s }}\right)$ and relayed packet $\left(\mathrm{n}_{\text {relay,s}}\right)$. When the destination node receives the RTS, it firstly checks whether packets should be sent to the initial sender node. Then CTS is recorded and responded to the sender. Here, the responded time must be outside any handshake. Furthermore, it cannot keep silent. If the receiver has DPs sent to the initial nodes, it will respond CTS to inform the sender $n_{\text {relay,s }}$ and $n_{\text {new,s }}$. If $n_{S} \geq n_{R}$, the bidirectional data exchange happens. If the receiver node does not have data transmitted, both $n_{\text {relay,R }}$ and $n_{\text {new,R }}$ are set as zero. Therefore, bidirectional transmission protocol will become the traditional three-way handshake. For RABIC-MAC, the busy duration can be calculated and included in the RTS (CTS) to inform one-hop neighbors to avoid transmitting-receiving collisions. The main reason is that the sender-receiver pair knows the delay of one-hop neighbor nodes, and the time and the number of exchanged DPs, and then it can calculate expected busy duration, which is included in the RTS (CTS). If the neighbor nodes overhear the controlling packets, they will extract the busy time and compute their corresponding silent duration. Then it will defer the initialization any transmission to avoid collision with the ongoing transmission.

After receiving the CTS and $n_{R}>0$, the initial sender nodes will make the transmission plan based on the CTS. In our protocol a new type of controlling packets 'NTF' are introduced. The main function of NTF is to renew the neighbor nodes' silent duration. As shown in Fig.1 (a), (b) and (c), respectively, the sender nodes' one-hop neighbors should receive the NTF to extend their silent duration so that the sender nodes can successfully receive the incoming bidirectional DPs. After transmitting the NTF, the senders will transmit their DPs. After transmitting the CTS, the receivers will wait for the duration of $\tau_{S, R}+T_{N T F}$ before they start to transmit their DPs. Here $T_{\text {guard }}$ is inserted in order to accommodate the slight error.

The busy duration is mainly set to inform the one-hop neighbor nodes of S-R nodes so that they can defer their transmission. This is a vulnerable period, so that any node sending a packet disturbs the bidirectional transmission. The busy duration of the S-R node pair can be computed by

$$
\left\{\begin{array}{l}
d_{\text {busy }, R T S}=2 \tau_{S, R}+T_{C T S}+T_{N T F}+\tau_{\max , S} \\
d_{\text {busy }, C T S}=\tau_{S, R}+d_{S P}+T_{N T F}+d_{\text {buss }, R}^{r x} \\
d_{\text {busy }, N T F}=d_{S P}+d_{\text {toc }, S}
\end{array}\right.
$$

After overhearing a controlling package and extracting its busy time, each node can calculate its local silent duration. The silent duration is not necessarily equal to the busy duration because of the long propagation delay. Each neighbor node allows transmitting the DP at the end of silence duration, so that the current transmission is not interfered by other transmitted packets. Therefore, the silent duration are given as followed.

$$
\left\{\begin{array}{l}
d_{\text {silent }, i}^{\text {RTS }}=d_{\text {bus }, R T S}-\tau_{s, i}, i \in N_{s} \\
d_{\text {sils }}^{\text {RIs }, j}=d_{\text {busy }, C T S}-2 \tau_{R, j}, j \in N_{R} \\
d_{\text {silent }, i}^{\text {NTF }}=d_{\text {bus }, N T F}-2 \tau_{R, j}, i \in N_{s}
\end{array}\right.
$$

$d_{\text {silent,i }}^{R T S}$ should be large enough so that all the neighbor nodes can extend themselves by overhearing NTF packet. The bidirectional transmission can be divided into three scenarios including the type 1, type 2 and type 3 . Here, these definitions such as SP, CR, RR are introduced as followed. Def.1: Special Round (SP) is defined as the first round. In this round, the number of DPs exchanged by sender and receiver is written as ksp. Def.2: A Complete Round (CR) is defined as a maximum number of DPs transmitted by both sender and receiver in the bidirectional transmission process. Def.3: A Residual Round (RR) is defined as the number of transmitted DPs owned by only the sender during the transmission or the number of transmitted DPs from receiver to sender, which is less than $\mathrm{k}_{\max }$. 
$\mathrm{T}_{\text {guard }}$ is introduced when the node switches transmitting and receiving modes to avoid any estimated delay error for the internal node transmission, the transceiver's TX-RX turnaround time and the swing of distance sensor caused by the fluctuation of water. To enhance the efficiency, the time for bidirectional transmission of DPs should be as short as possible. $d_{t o c, i}$ is given as

$$
d_{\text {toc }, i}=n_{C R} d_{C R}+d_{R R}, i \in\{S, R\}
$$

For the SP, CR and RR period, concurrent bidirectional data are transmitted in order to avoid data collision. The protocol should meet the following conditions.

$$
k_{i} T_{D A T A}+T_{\text {guard }} \leq \tau_{S, R}, i \in\{S, R\}
$$

From the formula (4), DPs can be calculated and transmitted in a round. The maximum of $\mathrm{k}_{\mathrm{i}}$ is

$$
k_{\max }=\left(\tau_{S, R}-T_{\text {guard }}\right) / T_{\text {DATA }}
$$

To pack the transmissions as tightly as possible, $k_{i}=k_{\max }$ should be satisfied in the CR cycles. $S-R$ pair nodes in three scenarios have the same cycle time CR, $d_{C R}=\tau_{S, R}+k_{\max } \times T_{D A T A}$. From Fig. 1 , when $\mathrm{k}_{\max }=2$, the number of $\mathrm{CR}$ can be computed by the following formula.

$$
n_{C R}=\left\lfloor\min \left(\alpha_{S}, \alpha_{R}\right)\right\rfloor
$$

where $\alpha_{i}=\left(n_{i}-k_{S P, \text { max }}\right) / k_{\text {max }}, i \in\{S, R\}$. If $n_{R} \leq n_{S}, \mathrm{n}_{C R}$ is reduced to $n_{C R}=\left(n_{R}-k_{S P, \max }\right) / k_{\max }$.

The sender node immediately transmits its DPs after transmitting NTF. However, the receiver node waits for the time after transmitting its CTS, and then starts to transmit its DPs. In this event transmitted $\tau_{S, R}+T_{N T F}$ DPs are defined as $d_{S P}$, where the maximum number of DPs is $k_{S P \text {, max }}$ in SP, which is expressed as

$$
k_{S P, \max }=\left\lfloor\frac{\tau_{S, R}-T_{\text {guard }}-T_{N T F}}{T_{D A T A}}\right\rfloor
$$

Here, we assume $k_{S P, \max }=1$. There are three different scenarios arising in RABIC-MAC

Type1: It is characterized that one SP, at least one CR and no $\mathrm{RR}$, then $n_{\mathrm{S}}=n_{R}$, and the completion time of its busy duration can be expressed as

$$
d_{\text {toc, } i}=d_{\text {bus }, i}^{r x}=n_{C R} d_{C R}+\left(n_{C R}-1\right) T_{\text {guard }}, i \in\{S, R\} .
$$

Type2: It is characterized that one SP, at least one CR and one RR, then $n_{S}>n_{R}$. In the RR, the sender node unidirectionally transmits its remaining DPs, and then its busy duration and the completion time can be expressed as:

$$
\left\{\begin{array}{l}
d_{\text {bus }, S}^{r x}=n_{C R}\left(d_{C R}+T_{\text {guard }}\right) \\
d_{\text {toc }, S}=d_{\text {busy }, S}^{r x}+\left(n_{S}-k_{s p, \max }-n_{C R} k_{\max }\right) T_{D A T A} \\
d_{t o c, R}=d_{\text {bus }, R}^{r x}=d_{t o c, S}+\tau_{S, R}
\end{array}\right.
$$

Type3: Different from the scenario 2, it is characterized by one SP, the optional number of CR and a single RR. Here, RR is further divided into two rounds including a mandatory RR1 and an optional RR2. Therefore, the bidirectional data transmission has several situations. (1) CR, RR1, RR2. (2) CR, RR1. (3) RR1, RR2. (4) RR1. RR2 condition exists only when $n_{S}>\left(n_{C R}+1\right) k_{\max }$. It is noteworthy that the receiver node transmits the number of $k_{R}<k_{\max }$ DPs in RR1. Once the sender node finishes receiving its DPs in RR1, it will transmit the remaining redundant packets unidirectionally in RR2. Here, we assume $n_{S}>n_{R}$. And its completion time, busy duration may be expressed as 


$$
\begin{aligned}
& d_{\text {busy }, S}^{r x}=n_{C R}\left(d_{C R}+T_{\text {guard }}\right)+\left(n_{R}-k_{S P, \max }-n_{C R} k_{\max }\right) T_{D A T A}+\tau_{S, R} \\
& d_{\text {toc }, S}=\left\{\begin{array}{l}
d_{b u s y, S}^{\text {rx }}+\left(n_{S}-k_{S P, \max }-\left(n_{C R}+1\right) k_{\max }\right) T_{D A T A}+T_{\text {guard }}, \quad \text { if RR is present } \\
d_{b u s y, S}^{r x}, \quad \text { otherwise }
\end{array}\right. \\
& d_{\text {toc }, R}=d_{\text {buss }, R}^{r x}=\left\{\begin{array}{l}
d_{t o c, S}+\tau_{S, R}, \quad \text { if RR is present } \\
n_{C R}\left(d_{C R}+T_{\text {guard }}\right)+\left(n_{S}-k_{S P, \text { max }}-n_{C R} k_{\text {max }}\right) T_{D A T A}+\tau_{S, R}, \quad \text { otherwise }
\end{array}\right.
\end{aligned}
$$

Each node is allowed to transmit at most $S_{\text {burst }}$ packets in a handshake, so the delay is shorter. If the sender and receiver nodes are too close, namely $\tau_{S, R}<T_{\text {DATA }}+T_{\text {guard }}$, then performances of the unidirectional transmission are better than bidirectional transmission. The main reason is that the communication overhead of the former is smaller than the latter. In the reserved phase of channels, a CTS packet may undergo collision with the intend receiver. It means that if the CTS packet is not successfully transmitted to sender in the period of $d_{S P}$, the sender node will not transmit DPs to the receiver node. However, the receiver node will transmit DPs to the sender node within the agreed time. Therefore, they cannot initiate its bidirectional data transmissions. To some extent, this will lead to low channel utilization. In this paper, we adopt the receiver node detecting mechanism. If receiver node detects that there is no incoming DPs from sender node in the period time of $d_{S P}$, it will assume a CTS failure. Therefore, they release channel at the end of the $d_{S P}$ and the sender node will be released from current handshake after its waiting-for-CTS (WFCTS) timer expiry. At last sender node restarts content the channel.

Here the RTS-triggering mechanism and backoff algorithm, namely the mixed mechanism of batch-by-size and batch-by-time, is used to determine the time when a RTS is triggered. A node triggers the RTS after the sender node accumulates at least $S_{\text {burst }}$ packets for one neighbor or after a sender initiates an RTS attempt if $T_{\max }$ has already elapsed since the release from previous handshake and at least one packet has been accumulated. In addition, to satisfy the above two conditions, it is also ensured that nodes are not required by other nodes to remain silent duration and currently not in any other handshake. Otherwise, it will delay RTS until its condition is satisfied. If a RTS fails in the competition, the node resets the backoff counter, its minimum value for $\mathrm{T}_{\mathrm{bk}}$. Finally the backoff interval can be expressed as

$$
T_{b k}=\text { uniform }\left\{0, B_{c n t}\right\} \times \tau_{\max }
$$

where $B_{c n t}$ is a window content, $\tau_{\max }$ is the max propagation delay to meet either of two RTS trigger conditions. An idle node will initialize its contention timer according to $\mathrm{T}_{\mathrm{bk}}$. Only if its timer expire reaches, RTS is transmitted.

\section{Simulation results}

The multi-hops topology is shown in Fig. 2, where 36 static nodes with the grid specie of $2000 \mathrm{~m}$ are assumed in the area. The maximum communication range is $3500 \mathrm{~m}$. Each node has exactly 8 one-hop neighbors and 16 second-hop neighbors. The channel is assumed to have no error so that the packet loss is only caused by collisions. Each node is equipped as a half-duplex omnidirectional transceiver. The underwater propagation speed is set as $1500 \mathrm{~m} / \mathrm{s}$ and the transmission is set as 4800 b/s. DPs are $1200 \mathrm{~b}$. The lengths of the RTS, CTS, and NTF are set as 152, 200, 136 and $72 \mathrm{~b}$, respectively. Here setting $T_{\text {guard }}=10 \mathrm{~ms}, B_{\min }=1$ and $B_{\max }=32$. Each node maintains two buffers for its one-hop neighbors. Each buffer may involve 100 packets. If RTS fails to attempt, it may attempt at last time, so a retransmission limit will be not designed. To avoid any transient effect, the simulated results are collected only from $2 \times 10^{4}$ to $1 \times 10^{5} \mathrm{~s}$. All results are averaged over 20 simulations. 


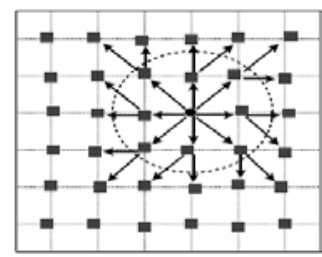

Fig.2. The multi-hop network topology

Throughput per node and the end-to-end delay based on the RABIC-MAC protocol are calculated, and then are compared with the BIC protocol. The throughput per node is defined as

$$
\gamma=\frac{1}{36}\left[\frac{\text { No. of packets received } \times \text { Packet length }}{\text { Transmission rate } \times \text { Simulation duration }}\right]
$$

The End-to-end data delay is defined as the duration when packets are transmitted successfully from source nodes to their destination. The variations of total throughput and the end-to-end delay with the normalized load per node are shown in Fig.3 (a) and (b), respectively.

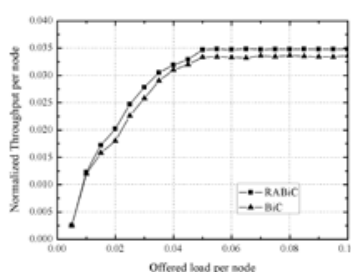

(a)

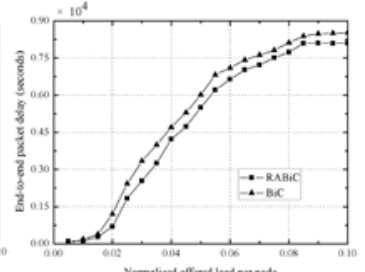

(b)

Fig.3 Variations of Throughput per node (a) and End-to-End packet delay (b) with various scheme

From Fig.3, the RABIC protocol evidently has higher saturated throughput and lower saturated end-to-end delay than the BIC protocol. If the offered load per node is lower than 0.02 , the throughput of BIC and RABIC are very close. However, if it is larger than 0.05 , the throughput of RABIC is better $2 \%$ than BIC. The main reason is that silence duration of one-hop neighbors is shortened, and then the neighbor nodes can themselves handshake trigger as fast as possible. On the other hand, the RABIC protocol also transmits DPs $\tau_{S, R}$ earlier than BIC. Generally, $\tau_{S, R}$ is mainly decided by the communication distance. For probing the influence of communication distance on the total throughput and the end-to-end delay, the communication distance is set as 4200 and $6125 \mathrm{~m}$, respectively. In underwater acoustic network, the way of signal attenuation and loss is different from terrestrial wireless. The attenuation of acoustic wave, denoted as $A(l, f)$, is affected by the propagation distance $l$ and the acoustic frequency $f$. $A(l, f)$ may be formulated from the following equation

$$
A(l, f)=l^{k} \times \alpha(f)^{l}
$$

Where $k$ is the energy spreading factor, which is set as 1 for cylindrically spreading and 2 for spherically spreading. $\alpha(f)$ is the absorption coefficient and dependent on frequency $f(\mathrm{kHz})^{[12]}$. Here

$$
10 \log \alpha(f)=\frac{0.11 f^{2}}{1+f^{2}}+\frac{44 f^{2}}{4100+f^{2}}+2.75 \times 10^{-4} f^{2}+0.003
$$

From formula (13) and (14), the farther the distance between nodes, the stronger signal attenuation and loss. Variations of total throughput (a) and the end-to-End delay (b) of UWANs with scheme for the distances 4200 and $6125 \mathrm{~m}$ are calculated and shown in Fig.4. 


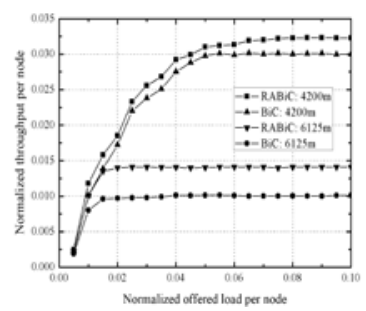

(a)

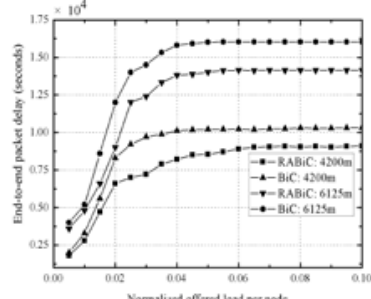

(b)

Fig.4 Variations of the throughput per node (a) and End-to-End packet delay (b) with various scheme

From Fig.4, the total throughput decreases and the End-to-End delay increases with the communication distance in that the signal attenuation and loss increases. More DPs can be transmitted in advance due to lager $\tau_{S, R}$. For $4200 \mathrm{~m}$, the throughput and the end to end delay are 0.035 and 8700s. They are improved $14 \%$ and $13 \%$ compared with BIC after they are stable. For $6125 \mathrm{~m}$, the throughput and end to end packet delay are 0.0145 and $13000 \mathrm{~s}$. They are improved 33\% and $23 \%$, compared with BIC. The increase rate for $6125 \mathrm{~m}$ is obviously higher than $4200 \mathrm{~m}$.

In conclusion, compared with the protocol BIC, the new protocol RABIC has higher channel utilization and lower end-to-end delay. Furthermore, RABIC is evidently better than BIC for improving the saturated throughput and the saturated end-to-end delay, especially, in lager communications range environment.

\section{References}

[1] M. Erol-Kantarci, H. T. Mouftah and S. Oktug: Communications Surveys \& Tutorials IEEE Vol. 13(2011), p. 487

[2] L. Hong, F. Hong, Z. Guo et al: Sensors Vol. 11(2011), p. 2920

[3] W. H. Liao, C. C. Huang: Sensors Journal IEEE Vol. 12 (2012), p.1686

[4] E. M. Sozer, M. Stojanovic, J. G. Proakis: Oceanic Engineering IEEE Journal Vol. 25 (2000), p. 72

[5] N. Chirdchoo, W. S. Soh, K. C. Chua: VTC Spring 2008. IEEE. IEEE (2008), P.46

[6] N. Chirdchoo, W. S. Soh and Chua K. RIPT: IEEE Journal on, 26(2008), p.1744

[7] W. H. Liao, Y. C. Lin and S. C. Kuai: 2014 International Conference on. IEEE, 2014. p.1

[8] H. H. Ng, W. S. Soh and M. Motani: Computer Networks, 57(2013), p. 2733

[9] J. Yang, P. Guo, T. Jiang et al: 2012 IEEE International Conference on. IEEE, 2012, p. 435-439.

[10]C. M. Chao, Y. Z. Wang and M. W. Lu: Systems, Man, and Cybernetics: Systems, IEEE Transactions on Vol. 43(2013), p. 128.

[11]H. H. Ng, W. S. Soh and M. Motani: Oceanic Engineering, IEEE Journal of Vol. 38(2013), p. 547

[12]L. M. Brekhovskikh and I. U. P. Lysanov: Springer Science \& Business Media, 2003. 